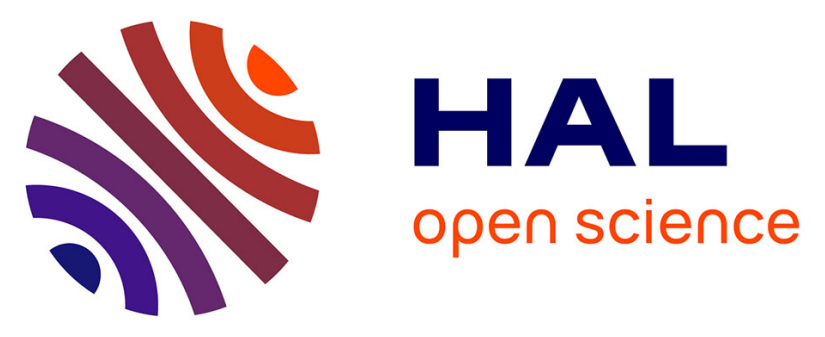

\title{
ShearWave TM Elastography A new real time imaging mode for assessing quantitatively soft tissue viscoelasticity
}

J Bercoff, A Criton, C C Bacrie, J Souquet, M Tanter, Jean Luc Gennisson, T Deffieux, M Fink, Valerie Juhan, Anne Colavolpe, et al.

\section{To cite this version:}

J Bercoff, A Criton, C C Bacrie, J Souquet, M Tanter, et al.. ShearWave TM Elastography A new real time imaging mode for assessing quantitatively soft tissue viscoelasticity. 2008 IEEE Ultrasonics Symposium, IEEE, Nov 2008, Beijing, China. 10.1109/ULTSYM.2008.0079 . hal-03275099

\section{HAL Id: hal-03275099 https://hal.science/hal-03275099}

Submitted on 30 Jun 2021

HAL is a multi-disciplinary open access archive for the deposit and dissemination of scientific research documents, whether they are published or not. The documents may come from teaching and research institutions in France or abroad, or from public or private research centers.
L'archive ouverte pluridisciplinaire $\mathbf{H A L}$, est destinée au dépôt et à la diffusion de documents scientifiques de niveau recherche, publiés ou non, émanant des établissements d'enseignement et de recherche français ou étrangers, des laboratoires publics ou privés. 


\section{ShearWave ${ }^{\mathrm{TM}}$ Elastography}

\section{A new real time imaging mode for assessing quantitatively soft tissue viscoelasticity}

\author{
J. Bercoff, A. Criton, C.C Bacrie, J. Souquet \\ SuperSonic Imagine \\ Aix en Provence, France \\ jeremy.bercoff@supersonicimagine.fr
}

\author{
M. Tanter, J.L. Gennisson, T. Deffieux, M. Fink \\ Laboratoire Ondes et Acoustique \\ ESPCI \\ Paris, France
}

\author{
Valerie Juhan, Anne Colavolpe \\ Hopital La timone \\ Marseille, France
}

\author{
Dominique Amy \\ Cabinet de Radiologie Amy-Fabry \\ Aix en Provence, France
}

\author{
Alexandra Athanasiou \\ Institut Curie \\ Paris, France
}

\begin{abstract}
ShearWave ${ }^{\mathrm{TM}}$ Elastography (SWE) is a new real time ultrasound imaging mode that quantitatively measures local tissue elasticity in kPa. Based on the Supersonic Shear Imaging concept (developped at the Laboratoire Ondes et Acoustique, Paris), this new concept may appear as a promising tool to improve breast lesion characterization. In vitro experimental measurements have been performed to quantify SWE mode performances in terms of resolution, penetration and the ability to measure quantitatively elasticity. Results show that the SWE mode exhibits a millimetric resolution and quantifies properly tissue elasticity on a wide range of elastic contrasts (from 7 to 110 $\mathrm{kPa}$ ). The real time capabilities and the robustness of the mode have been tested in clinical conditions, on breast lesions. 150 patients have been scanned with SWE mode in three different sites. Results show that SWE performs well on breast pathologies and presents a very good inter-site reproducibility. Finally, the quantitative elasticity value was analyzed as a function of pathology using FNA or core biopsy as the reference diagnostic method.
\end{abstract}

Keyword: shear wave, elastography, Mach cone, supersonic, transient, quantitative, $\mathrm{kPa}$, elasticity, viscosity.

\section{INTRODUCTION}

Elastography techniques aim to assess tissue differences in stiffness and provide an imaging representation of what was historically assessed qualitatively by palpation. The main objectives of Elastography are to improve diagnostic confidence, and increase specificity of the ultrasound exam.

Static elastography is today available on many ultrasound systems. It uses a uniform compression at the surface of the body to cause deformation of the tissue. The compression is applied by the user and the ultrasound scanner calculates and displays the induced deformation in the imaging plane $[1,2]$. Young's modulus cannot be reconstructed as the stress induced is unknown within the tissues. The clinical relevance of the static elastography has been extensively studied. While promising results have been demonstrated, many pitfalls have been reported by the users including poor reproducibility, inter-operator variability and lack of quantitative information [3-6]. Dynamic elastography utilizes a continuous (monochromatic) vibration. Stationary waves induced in the body are analyzed to deduce tissue elasticity [7,8]. Dynamic elastography is well suited for MR systems as the vibration pattern is not time dependent and must be assessed in a volume. It is a quantitative approach but suffers from the usual MR drawbacks: high cost, limited availability, and lack of real time imaging. Shear wave based elastography utilizes transient pulses to generate propagating shear waves in the body [9-11]. The elasticity is directly deduced by measuring the wave propagation speed. Shear wave based elastography is the only approach able to provide quantitative and local elastic information [12] in real time. However, its implementation as a real time ultrasound imaging mode requires major technological breakthroughs in ultrasound system architecture. Aixplorer ${ }^{\mathrm{TM}}$, the ultrasound system developed by SuperSonic Imagine, is the first ultrasound system to leverage this technology and implement a true shear wave-based elastography mode (ShearWave ${ }^{\mathrm{TM}}$ Elastography - SWE).

This study presents a first assessment of the technical performance of this new imaging concept (contrast, resolution, robustness), highlights its applicability in clinical routine, and analyzes its features in the breast imaging workflow.

\section{System AND Mode PRESEntation}

SWE mode displays elasticity using a color coded image superimposed on the top of the B-mode image. The color scale is quantitative with values expressed in $\mathrm{kPa}$. Stiffer tissues are coded in red and softer tissues in blue.

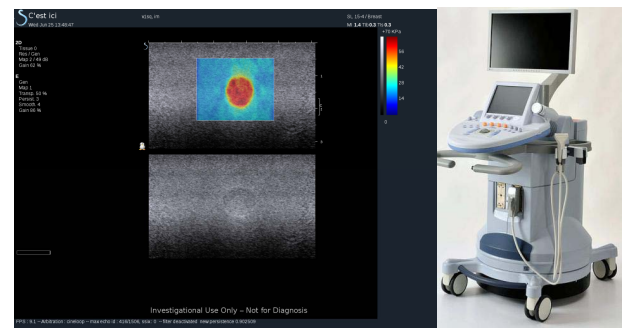

Figure 1: SWE mode overview (left), Aixplorer ${ }^{\mathrm{TM}}$ system (right) 
An example is shown on figure 1 and corresponds to an elasticity map of a phantom containing a harder inclusion. The elasticity image is refreshed in real time. The imaging frame rate is optimized to meet international standards acoustic output limitations [13]. To compute elasticity maps, SWE mode generates transient shear waves and captures their propagation using the Supersonic Shear Imaging (SSI) method [11]:

- Generation of shear waves is performed using supersonic shear wave sources that are created thanks to the acoustic radiation force of ultrasound focused beams [14]. In a supersonic regime, shear waves are coherently summed in a "Mach cone" formation, thus increasing their amplitude and improving their propagation distance. For a fixed acoustic power at a given location, this method increases shear wave generation efficiency by a factor 4 to 8 compare to a non supersonic source.

- Transient shear wave propagation is captured using ultrafast imaging. A set of flat insonifications are performed at a very high frame rate $(3000$ to $8000 \mathrm{~Hz})$ in order to image the medium during shear waves propagation.

Elasticity maps of the medium are deduced from equation (1) after having measured shear wave propagation velocity at each location in the imaging plane.

This is achieved using a linear ultrasound probe without requiring any intervention by the user.

$$
E=3 \rho c^{2}(1)
$$

Supersonic Imagine's system, Aixplorer ${ }^{\mathrm{TM}}$, is the first ultrasound system able to, firstly, reach such ultrafast frame rates of thousands of $\mathrm{Hz}$ and, secondly, perform ShearWave elastography sequences. The system architecture is entirely software based: the digitized per channel RF Data are transferred at very high data rates via a PCI Express bus to a computer that processes data in real time (up to 5 GBytes/s). Aixplorer ${ }^{\mathrm{TM}}$ computes all the lines of each image in parallel, and therefore manages to process images acquired at ultrafast frame rates of thousands of $\mathrm{Hz}$. Its processing capabilities allow calculation of an elasticity map in less than a tenth of a second.

To compute a full elasticity map as displayed on the system screen (figure 1), several supersonic radiation force lines (at least 2) are generated. For each line, elasticity maps are calculated and then combined in a final one. The real time capabilities of the mode are intrinsically linked to the efficiency of the supersonic shear wave generation as the number of pushing beams necessary to compute a full elasticity map can be limited to a small number, allowing the acoustic power necessary to perform elasticity images to remain within international standards limitations.

\section{SWE MODE PERFORMANCE ASSESSMENT}

Performances of the new elasticity imaging mode has been performed on custom calibrated phantoms and illustrated on clinical cases. Four parameters have been evaluated: 1) the ability of the mode to correctly measure the true elasticity (in $\mathrm{kPa}$ ) of purely elastic media, 2) resolution, 3) the ability to locally measure tissue elasticity, without suffering from interference from the surrounding tissues, 4) reproducibility.

\section{A. Quantitative aspect}

The ability of the mode to quantitatively measure tissue elasticity has been studied on a set of 5 calibrated cubic phantoms having typical elasticity values encountered in breasts [15]. Phantoms have been provided by University of Wisconsin where reference elasticity has been measured at 50 $\mathrm{Hz}$ using a rheometer [16]. Elasticity values measured with SWE has been performed by taking the average value and standard deviation of a $6 \mathrm{~mm}^{2}$ box centered on the elasticity image.

\begin{tabular}{|c|c|c|c|c|c|}
\hline & Medium 1 & Medium 2 & Me dium 3 & Medium 4 & Medium 5 \\
\hline $\begin{array}{c}\text { Reference } \\
\text { elasticity }\end{array}$ & 14 & 20 & 37 & 72 & 105 \\
$\begin{array}{c}\text { Measured } \\
\text { elasticity }\end{array}$ & $\mathbf{1 5 . 0 8}$ & 21.3 & 37.4 & 74.7 & 105.7 \\
\hline Stdl & 2.3 & 3.1 & 5.4 & 9.6 & 11.5 \\
\hline
\end{tabular}

Table 1: Elasticity measurements on different calibrated phantoms.

Table 1 shows very good agreement between the two measurement methods demonstrating the quantitative aspect of the SWE mode.

\section{B. Resolution}

Resolution has been assessed using a parallelipedic phantom containing layers of different elasticities. Axial and lateral resolutions have been measured by imaging horizontal and vertical interfaces using SWE mode. The elastography image of the interface can be considered as a slope centered on the true interface. The model used to quantify that slope is an error function (erf) as described on equations below:

$$
\begin{aligned}
& E_{\text {real }}=E_{1}+\left(E_{2}-E_{1}\right) \cdot H\left(x-x_{i}\right) \\
& E_{\text {estimated }}=E_{1}+\frac{\left(E_{2}-E_{1}\right)}{2} \operatorname{erf}\left(\frac{x-x_{i}}{\sigma}\right)
\end{aligned}
$$

$\sigma$ is defined as the resolution of the mode for the considered elasticity contrast $\left(E_{2}-E_{1}\right)$ and is estimated by fitting the experimental data with the model function. Resolution measurements have been performed for different elasticity contrasts and are shown below.

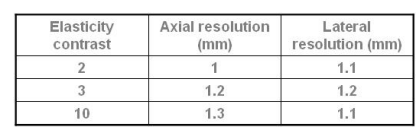

Table 2: Resolution of SWE mode

Results show that SWE provides a millimetric resolution that remains quasi-constant for all elasticity contrasts available.
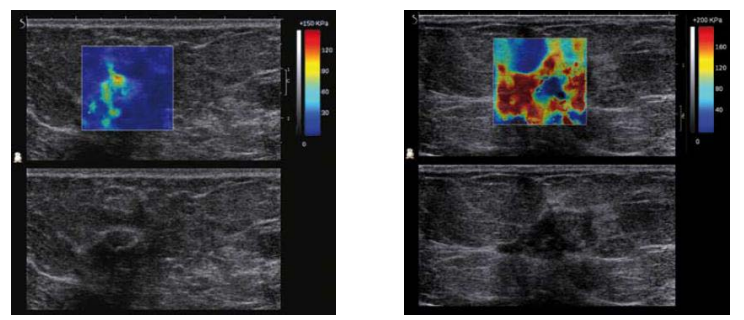

Figure 2: Elasticity image of a millimetric lesion (left) and of a complex lesion (right). 
The ability of the mode to quantify elasticity of millimetric lesion is illustrated figure 2 (right). A $6 \mathrm{~mm}$ lesion presents a localized elasticity of $130 \mathrm{kPa}$.

\section{C. $\quad$ Local aspect of elasticity measurement}

The local aspect of the elasticity estimation is one of the main features that SWE offers the clinician compared to static elastography techniques. The strain induced in tissue by an external compression is not only operator dependent, but also tissue dependent. Under the same stress, a soft tissue area will be deformed differently depending on the presence and relative position of a hard area in its proximity. In contrast, the shear wave propagation speed relates directly to tissue local elasticity. This allows SWE mode to provide local elasticity estimation with millimetric resolution. This is illustrated on the figure below where a hard lesion with a liquid center in a homogeneous tissue mimicking phantom is scanned in both SWE and static elastography mode. On the SWE image (left image) a red (representing harder tissue) couronne can be clearly seen with a center with no elasticity value which corresponds to the liquid area, while on the static elastography image (on the right) the whole lesion is colored in blue (representing hard) even in the liquid center..

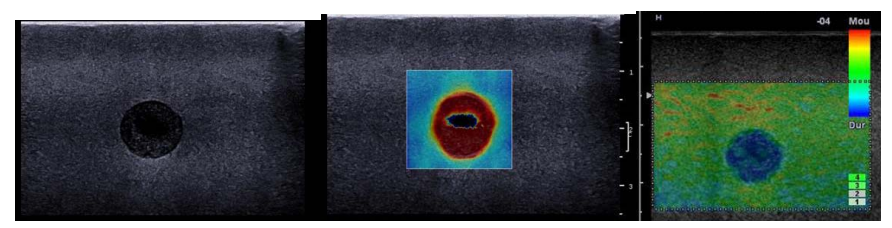

Figure 3: Phantom containing a cystic lesion within a harder shell using SWE (left) and static approaches (right)

SWE can help the radiologist to better understand tissue or pathologic morphology as illustrated on figure 2 (right). This lesion, classified as ACR5 using ultrasound BI-RADS ${ }^{\odot}$ lexicon, shows a hypoechoic center and heterogeneous borders on the ultrasound image. The elasticity image presents a hard shell (of more than $200 \mathrm{kPa}$ ) slightly larger than the size of the lesion on the B-mode. The shell is limited on the top left of the image by a fat lobe presenting very clear ultrasound and elasticity signatures (soft $\sim 30 \mathrm{kPa}$ ). The lesion has a soft center, information that could not be assessed using static approaches. A biopsy has been performed in the lesion center indicating presence of necrotic cells.

\section{Reproducibility}

Reproducibility of the method has been tested in in vivo breast scans. The main parameter that could alter reproducibility of the mode is variation in the compression level applied on the breast when scanning. As breast is non linear viscoelastic medium, compression changes elastic properties. This is illustrated on the following experiment where a physician was asked to apply five different level of compression while performing SWE mode. Elasticity values measured by the mode are displayed on figure 5: The hardening effect is low for slight compressions but became significant for the two strongest levels of compression. However one should note that those strongest levels of compression induced pain to the patient and would normally never be reached during a normal scan.

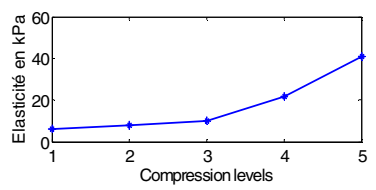

Figure 4: influence of different level of compression on breast tissue elasticity

To verify this, reproducibility tests were conducted on healthy breasts. Patients were asked to breath normally and the physician was asked to scan as usual. 15 scans were performed on the same breast cut (10 o'clock, $5 \mathrm{~mm}$ from the nipple). After each SWE scan, the physician was asked to release the probe and wait a few minutes before restarting the next SWE scan. Elasticity was measured in glandular tissue and in fat for each scan and the results are shown below.

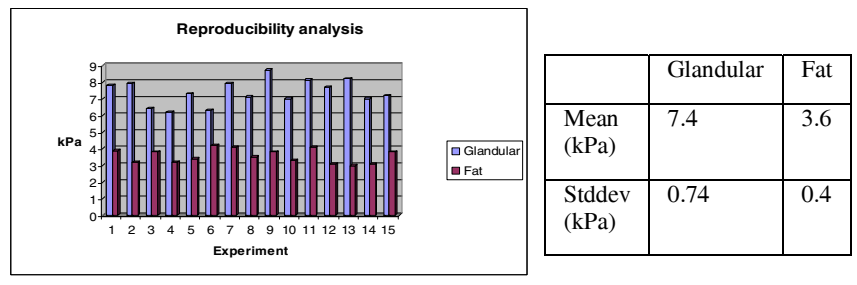

Figure 5: Reproducibility analys is

Results show a good reproducibility of the SWE mode in real scanning conditions.

\section{SWE MODE IN THE BREAST IMAGING WORKFLOW}

\section{A. Methods}

\section{Protocol}

After approval of the clinical study by French Competent Authority, 173 patients have been scanned using SWE mode among four different clinical sites: three hospitals and one private clinic. Patients first undergo a classical ultrasound examination on a conventional ultrasound system leading to a BI-RADS $^{\odot}$ score. Then a SWE scan was performed and the lesion elasticity was measured All women presenting scores higher than 3 were sent for a biopsy. Biopsy results were used as a reference when available. For BI-RADS ${ }^{\oplus} 2$ and some BIRADS $^{\odot} 3$ lesions (non biopsied), the reference was the ultrasound exam.

Elasticity measurement methodology

For each case, the quantitative elasticity of the lesion was measured according to the following rule: Lesion elasticity (E) is the mean value of a $5 \mathrm{~mm}^{2}$ box centered on the maximum elasticity value found within the echographic signature of the lesion.

Data Analysis

Correlation of elasticity distribution among sites has been analyzed and considered as a good representation of the technique operator independency of this technique.

Distribution of elasticity has been analyzed and plotted as a function of pathology. 


\section{B. Results}

\section{Inter-site data analysis}

Correlation of the elasticity data distribution between sites has been analyzed to estimate operator independency of the technique. Figure 6 shows box and whisker plot of the distribution among different sites. Table below shows mean, standard deviation of each distribution and comparison two by two using Mann Whitney test.

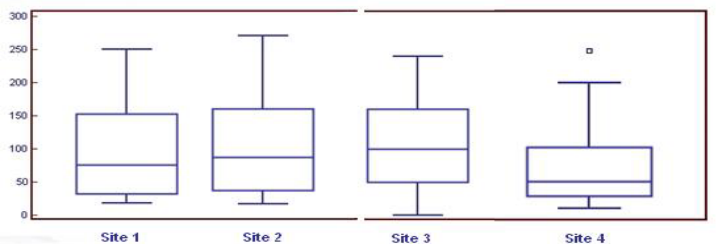

\begin{tabular}{|l|l|l|l|l|}
\hline & Site 1 & Site 2 & Site 3 & Site 4 \\
\hline Number of lesions & 41 & 40 & 69 & 33 \\
\hline malignant - benign ratio & 0.84 & 0.86 & 0.84 & 0.57 \\
\hline Mean & 98 & 104 & 103 & 77 \\
\hline Std & 71 & 71 & 67 & 64 \\
\hline
\end{tabular}

Figure 6: inter-site analysis

Results show that there is an excellent agreement of the elasticity distribution among 3 clinical sites $(\mathrm{P}=0.94, \mathrm{P}=0.94$ and $\mathrm{P}=0.57$ using the Mann Whitney test). The last site presents less cases and a lower proportion of malignant lesions, explaining the shift of the distribution through lower elastic values. These preliminary results demonstrate the robustness of the technique and the reliability of the elasticity measurements across different sites.

Analysis of tissue elasticity information in the breast imaging workflow

Figure 7 (left) shows the BI-RADS ${ }^{\odot}$ classification of the lesions as a function of the pathology. No false positive or negative has been found for BI-RADS ${ }^{\odot} 2,3$ and 5. BI-RADS ${ }^{\odot}$ 4 cases present as many benign as malignant lesions. The same analysis is shown for breast elasticity values (right).

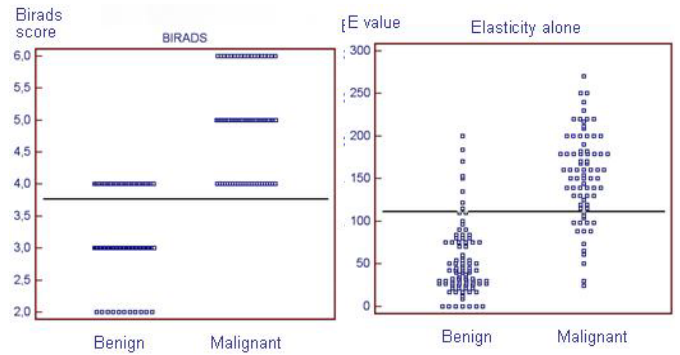

Figure 7: Distribution of ${\mathrm{BI}-\mathrm{RADS}^{\odot}}^{\odot}$ score and elasticity as a function of pathology.

Results show that SWE elasticity values of the majority of benign lesion are in the 1-70 $\mathrm{kPa}$ range. Hard benign lesions (8) correspond to fibroadenomas (2) and to scars (6). Elasticity values of malignant lesions are spread over a wider range going of 30 to $270 \mathrm{kPa}$ with a distribution mean around 160 $\mathrm{kPa}$. These results are consistent with literature data [15].

\section{CONCLUSION}

ShearWave ${ }^{\mathrm{TM}}$ Elastography is a new real-time ultrasound imaging concept that quantitatively measures local tissue elasticity in $\mathrm{kPa}$ with a millimetric resolution. The Aixplorer ${ }^{\mathrm{TM}}$ ultrasound system, developed by SuperSonic Imagine, is the only ultrasound scanner able to leverage that technology. The robustness and pertinence of the SWE mode have been analyzed for breast imaging on 173 patients. Results show that mode behaves reliably in clinical conditions, presents an excellent inter-site reproducibility and provides new quantitative information that physicians could use in their breast diagnostic workflow.

\section{REFERENCES}

[1] Ophir J, Céspedes I, Ponnekanti H, Yasdi Y, Li X. Elastography: A quantitative method for imaging the elasticity of biological tissues. Ultrason Imaging 1991;13:111-134.

[2] Hall TJ, Zhu Y, Spalding CS. In vivo real-time freehand palpation imaging. Ultrasound Med Biol 2003;29:427- 435.

[3] Locatelli M, Chervesani R, Rizzatto G. Real-time ultrasound Elastography: diagnostic tool or electronic gadget ? Eur Radiol 2005, supplement 1 to Vol 15, abstract B-0255, p 139.

[4] Svensson W.E., Amiras D: Ultrasound elasticity imaging. Breast Cancer Online 2006,9:e24:7pages. Cambridge University Press.

[5] Ako, Ei Ueno, Eriko, Hiroshi, Hideto, Tsuyoshi, Makoto, Takeshi. Breast Disease: Clinical Application of US Elastography for Diagnosis. Radiology 2006 Radiology; Volume 239.

[6] Tardivon A, El Khoury C, Thibault F, Wyler A, BarreauB, Neuenschwander S. Elastosonography of the breast: Prospective study of 122 lesions. J Radio 2007;88:657-62.

[7] Muthupillari R, Lomas DJ, Rossman PJ, Greenleaf JF, Manduca A, Ehman RL. Magnetic resonance elastography by direct visualization of propagating acoustic strain wave. Science 1995;269:1854-1856.

[8] Parker KJ, Lerner RM. Sonoelasticity of organs: Shear waves ring a bell. J Ultrasound Med 1992;11:387-392

[9] Sarvazyan AP, Rudenko OV, Swanson SD, Fowlkes JB, Emelianov SY. Shear wave elasticity imaging - A new ultrasonic technology of medical diagnostic. Ultrasound Med Biol 1998;20:1419-1436.

[10] Nightingale KR, McAleavey SA, Trahey G. E. Shear wave generation using acoustic radiation force: In vivo and ex vivo results. Ultrasound Med Biol 2003;29(2):1715-1723.

[11] J. Bercoff, M. Tanter, et M. Fink, "Supersonic shear imaging: a new technique for soft tissue elasticity mapping," IEEETrans. on ultrasonics, ferroelectrics, and frequency control, vol. 51, Avr. 2004, pp. 396-409.

[12] Tanter M, Bercoff J, Athanasiou A, Deffieux T, Gennisson JL, Montaldo G, Muller M, Tardivon A, Fink M. Quantitative Assessement Of Breast Lesion Viscoelasticity: Initial Clinical Results Using Supersonic Shear Imaging. Ultrasound Med.\&Biol., Vol. 34, No. 9, pp. 1373-1386, 2008.

[13] IEC 60601-2-37:Medical electrical equipment - Part 2-37: Particular requirements for the safety of ultrasonic medical diagnostic and monitoring equipment.

[14] Bercoff J, Tanter M, Fink M. Sonic boom in soft materials: The elastic Cerenkov effect. Appl Phys Lett 2004;84(12):2202-2204.

[15] Sarvazyan, A.P. (2001). Elastic Properties of Soft Tissue. Handbook of Elastic Properties of Solids, Liquids, and Gases. 3: 107-127.

[16] E. L. Madsen, M.A. Hobson, G. R. Frank, H Shi, J. Jiang, T. J. Hall, T. Varghese, M. M. Doyley and J. B. Weaver, Anthropomorphic breast phantoms for testing elastography systems. Ultrasound in Med. \& Biol., Vol. 32, No. 6, pp. 857-874, 2006 